\title{
Two-group Raman Optical Activity Revisited
}

\author{
David L. Andrews \\ School of Chemical Sciences, University of East Anglia, Norwich, UK NR4 7TJ
}

\begin{abstract}
In the circular differential Raman scattering observed in biological and other large polyfunctional molecules, many spectral features may be attributed to conferred chirality, in the following sense. Although a given vibrational transition may occur within a group in an intrinsically achiral local environment, it is coupling with another achiral but dissymmetrically placed group that generates the chiral response. Where two groups so coupled are chemically dissimilar, the effect originates in interference between one- and two-centre scattering mechanisms. The one-centre mechanism entails vibrational transition by a group as it undergoes conventional Raman scattering. The two-centre mechanism involves mediation of the chiral influence of a second group on this transition by Förster-type radiationless energy transfer. Where quantum-mechanical interference generates a differential Raman signal, the circular intensity differential depends on the inverse square of the distance between the groups so coupled. This distance dependence may be understood as originating from a combination of two factors. One is the linear distance dependence characterising Raman optical activity due to direct interference between transitions at distinct sites, which arises in the case of chemically identical groups. The other is the inverse cubic distance dependence associated with the probability amplitude for Förster energy migration. The Raman optical activity of any group with no chemical equivalents in its vicinity should thus be interpreted as resulting from a sum of inverse-square couplings with other chromophores. The two-group model for Raman optical activity is critically assessed, possible ways to improve upon the model are considered, and the result for the differential scattering intensity is recast in a new form that is more general and also more concise than has hitherto been presented.
\end{abstract}

\section{Introduction}

The coupling of two intrinsically achiral groups as a model of molecular chirality has origins that can be traced back over nearly 80 years. ${ }^{1-5}$ At first sight it appears surprising that, in spite of all the advances in molecular quantum calculations, such a basically crude model should nowadays retain any significant utility. Yet it does so, at least in part, because modern software remains largely unamenable to the calculation of chiral parameters for large polyatomic species. Moreover, although originally developed for the description of optical rotatory phenomena with an essentially electronic origin, the concept now finds important applications in the description of Raman optical activity (ROA). ${ }^{6-11}$

The application of a two-group model to chiral phenomena entailing molecular vibrations does have a certain logical appeal. In the vibrational spectrum of a chiral polyfunctional molecule it is generally easy enough to ascribe particular features to the vibrations of groups which, in terms of their local symmetry, are intrinsically achiral. When such spectral features prove to display a differential response to left and right 
circularly polarised light, an interpretation in terms of conferred chirality may be entertained, and the two-group model offers a direct insight into the mechanism for the conferral. The broad, though not exact, agreement that has been found between the results of two-group and $a b$ initio ROA calculations for small polyatomics is also encouraging. ${ }^{12}$

In this paper, the appropriation of a two-group model for ROA is critically reassessed and the result for the differential scattering intensity is recast in a new form that is more general and also more concise than has hitherto been presented. Possible ways to improve upon the model are discussed, and a number of new directions for the theory and its applications are considered.

\section{Reformulation of Two-group ROA}

The origin of chiral discrimination in the two-group model of ROA can perhaps best be understood in terms of interference between quantum probability amplitudes for distinct scattering mechanisms with common initial and final states. Specifically, we can entertain four different types of mechanism for scattering processes involving two groups A and $\mathrm{B}$. The four possibilities are associated with the annihilation of an incoming photon (wavevector $\boldsymbol{k}$, frequency $\omega=c|\boldsymbol{k}|$, circular polarisation vector $\boldsymbol{e}^{\mathbf{L} / \mathbf{R}}$ ) and the creation of a Raman-shifted photon (wavevector $\boldsymbol{k}^{\prime}$, frequency $\omega=c\left|\boldsymbol{k}^{\prime}\right|$, linear polarisation vector $\left.e^{\prime}\right)$ in each case at either A or B. Thus, without regard at this stage to the detailed structure and selection rules governing each term, we have an overall probability amplitude that can be expressed as:

$$
\begin{aligned}
& M=M_{\mathrm{AA}} \operatorname{exp~i~}\left(\boldsymbol{k} \cdot \boldsymbol{R}_{\mathrm{A}}-\boldsymbol{k}^{\prime} \cdot \boldsymbol{R}_{\mathrm{A}}\right)+M_{\mathrm{BB}} \operatorname{exp~i~}\left(\boldsymbol{k} \cdot \boldsymbol{R}_{\mathrm{B}}-\boldsymbol{k}^{\prime} \cdot \boldsymbol{R}_{\mathrm{B}}\right) \\
& +M_{\mathrm{AB}} \operatorname{exp~i~}\left(\boldsymbol{k} \cdot \boldsymbol{R}_{\mathrm{A}}-\boldsymbol{k}^{\prime} \cdot \boldsymbol{R}_{\mathrm{B}}\right) \\
& +M_{\mathrm{BA}} \operatorname{exp~i(}\left(\boldsymbol{k} \cdot \boldsymbol{R}_{\mathrm{B}}-\boldsymbol{k}^{\prime} \cdot \boldsymbol{R}_{\mathrm{A}}\right)
\end{aligned}
$$

where $\boldsymbol{R}_{\mathrm{A}}$ and $\boldsymbol{R}_{\mathrm{B}}$ denote the position vectors for groups $\mathrm{A}$ and $\mathrm{B}$, respectively, and $\boldsymbol{M}_{\mathrm{XY}}$ represents the net amplitude associated with photon incidence at $\mathrm{X}$ and emergence at $\mathrm{Y}$. Within the confines of an electric dipole approximation for each centre, the circular differential response arises purely from the interference terms, i.e.

$$
\begin{aligned}
\delta|M|^{2}= & 2 \mathscr{R}\left(M_{\mathrm{AA}} \bar{M}_{\mathrm{BB}} \exp (-\mathrm{i} \Delta \boldsymbol{k} \cdot \boldsymbol{R})+M_{\mathrm{AA}} \bar{M}_{\mathrm{AB}} \exp \left(\mathrm{i} \boldsymbol{k}^{\prime} \cdot \boldsymbol{R}\right)\right. \\
& +M_{\mathrm{AA}} \bar{M}_{\mathrm{BA}} \exp (-\mathrm{i} \boldsymbol{k} \cdot \boldsymbol{R}) \\
& +M_{\mathrm{BB}} \bar{M}_{\mathrm{AB}} \exp (\mathrm{i} \boldsymbol{k} \cdot \boldsymbol{R})+M_{\mathrm{BB}} \bar{M}_{\mathrm{BA}} \exp \left(-\mathrm{i} \boldsymbol{k}^{\prime} \cdot \boldsymbol{R}\right) \\
& +M_{\mathrm{AB}} \bar{M}_{\mathrm{BA}} \exp (-\mathrm{i} \Sigma \boldsymbol{k} \cdot \boldsymbol{R})
\end{aligned}
$$

where $\boldsymbol{R}=\boldsymbol{R}_{\mathrm{B}}-\boldsymbol{R}_{\mathrm{A}}, \Delta \boldsymbol{k}=\boldsymbol{k}-\boldsymbol{k}^{\prime}, \boldsymbol{\Sigma} \boldsymbol{k}=\boldsymbol{k}+\boldsymbol{k}^{\prime}$ and an overbar denotes complex conjugation. The similarity in structure of each term in eqn. (2) invites expression of the result as:

$$
\delta|M|^{2}=2 \mathscr{R} \sum_{n}\left(|M|^{2}\right)^{(n)} \exp \left(\mathrm{i} \boldsymbol{k}^{(n)} \cdot R\right)
$$

with $\boldsymbol{k}^{(1)}=-\Delta \boldsymbol{k}, \boldsymbol{k}^{(2)}=\boldsymbol{k}^{\prime}$ etc. It is clear that the extent of chiral discrimination depends on the magnitude of the group separation $R \equiv|R|$ relative to the inverse magnitude of the vectors $k^{(n)}$. This highlights the facility to model molecular chirality in terms of a local electric dipole (E1) approximation for each group; the system is essentially responsive to higher orders of the multipolar expansion (M1, E2 etc.) through the variation in the radiative electric fields between the two centres.

To calculate the detail of the chiral response we next need to introduce salient features of the radiation-matter coupling. Since each term in eqn. (1) entails tensor coup- 
ling with the radiation polarisation tensor $\overline{\mathbf{e}}^{\prime} \mathbf{e}^{\mathrm{L} / \mathrm{R}}$, the six terms in eqn. (2) and (3) all carry the radiation tensor $\overline{\boldsymbol{e}}^{\prime} \mathbf{e}^{\mathrm{L} / \mathbf{R}} \boldsymbol{e}^{\prime} \mathbf{e}^{\mathrm{L} / \mathbf{R}}$. The circular differential Raman scattering intensity can thus be written as:

$$
\Delta I^{(n)} \propto \mathscr{R}\left[\chi_{i j k l}^{(n)} e_{i}^{\prime} e_{j}^{\mathbf{R}} e_{k}^{\prime} \bar{e}_{l}^{\mathbf{R}} \exp \left(\mathrm{i} \boldsymbol{k}^{(n)} \cdot \boldsymbol{R}\right)-\chi_{i j k l}^{(n)} e_{i}^{\prime} e_{j}^{\mathrm{L}} e_{k}^{\prime} \bar{e}_{l}^{\mathrm{L}} \exp \left(\mathrm{i} \boldsymbol{k}^{(n)} \cdot \boldsymbol{R}\right)\right]
$$

where vector and tensor indices are referred to an arbitrary frame of reference and there is implied summation over repeated indices; detailed structures for each of the molecular response tensors $\chi_{i j k l}^{(n)}$ are given in the Appendix. Before proceeding further, it should be observed that eqn. (4) in its general form accommodates all orders of coupling (including where appropriate zero-order) between groups A and B, and is also written without necessary regard to either the normal exclusion of overlap between the group wavefunctions, or any assumption of index symmetry within the molecular response tensors of either group. Specifically, the latter relaxation allows the theory to accommodate resonance features.

Further progress is now expedited by the identity ${ }^{13}$

$$
e_{j}^{\mathrm{R}} \bar{e}_{l}^{\mathrm{R}}-e_{j}^{\mathrm{L}} \bar{e}_{l}^{\mathrm{L}}=\mathrm{i} \varepsilon_{j l m} \hat{k}_{m}
$$

where $\varepsilon_{j l m}$ is the Levi-Civita antisymmetric tensor. In the current context, the sensitivity of the quantity represented by eqn. (5) to molecular chirality is attested by the appearance on the right-hand side of the input wavevector direction. Naturally this cannot couple with either chromophore within the electric dipole approximation; it couples to the pair through their relative displacement vector. Referring the tensor components $\chi_{i j k l}^{(n)}$ to a molecular frame (denoted below by Greek indices) in which they are rotationinvariant, and bearing in mind the isotropic character of the Levi-Civita tensor, we now have

$$
\Delta I^{(n)} \propto-\mathscr{J} \chi_{\lambda \mu \nu \sigma}^{(n)} \varepsilon_{\mu o \pi} e_{i}^{\prime} e_{k}^{\prime} \hat{k}_{m}\left\langle l_{i \lambda} l_{k v} l_{m \pi} \exp \left(\mathrm{i} k_{n}^{(n)} R_{\rho} l_{n \rho}\right)\right\rangle
$$

Here the direction cosine $l_{i \lambda}$, for example, represents the $(i, \lambda)$ element of the Euler angle matrix that relates the laboratory and molecular frames, and the angular brackets denote the orientational averaging required for a fluid system.

Evaluation of the latter phased average is technically difficult. One approach has been to expand the exponential as a Taylor series; the zero-order term proves to yield a vanishing contribution to the right-hand side of eqn. (6) and the leading non-zero contribution then comes from the term linear in the exponent. However, the phased average can now be directly and exactly evaluated, through use of calculational techniques ${ }^{14}$ not available at the time of most earlier investigations, giving a result that corresponds to inclusion of all orders of the Taylor series expansion. Thus we find:

$$
\left\langle l_{i \lambda} l_{k v} l_{m \pi} \exp \left(\mathrm{i} k_{n}^{(n)} R_{\rho} l_{n \rho}\right)\right\rangle=\sum_{j=0}^{3} I_{i k m ; \lambda v \pi}^{(3 ; j) \phi}\left(\left|\boldsymbol{k}^{(n)}\right|, \hat{\boldsymbol{k}}^{(n)}, \hat{\boldsymbol{R}}\right)
$$

where each of the terms distinguished by $j$ values in the interval $(0,3)$ has a different tensor structure and carries a spherical Bessel function of the first kind, ${ }^{15} j_{j}^{(n)} \equiv j_{j}\left(k^{(n)} R\right)$ of order $j$. The $j=0$ and $j=2$ terms in the summation are real quantities and therefore make no contribution to eqn. (6); the result thus hinges on the $j=1$ and $j=3$ terms, which are pure imaginary. Explicitly, we obtain the following result:

$$
\begin{aligned}
\Delta I \propto \sum_{n}\left\{\left[\left(2 \beta \gamma^{(n)}-4 \alpha^{(n)}\right) j_{1}^{(n)}+\left(2 \beta \gamma^{(n)}+\alpha^{(n)}-5 \alpha^{(n)} \gamma^{(n)^{2}}\right) j_{3}^{(n)}\right] \chi_{\lambda \mu \lambda \nu}^{(n)} \varepsilon_{\mu v o} \hat{R}_{o}\right. \\
\quad+\left[\left(\alpha^{(n)}-3 \beta \gamma^{(n)}\right) j_{1}^{(n)}+\left(2 \beta \gamma^{(n)}+\alpha^{(n)}-5 \alpha^{(n)} \gamma^{(n)^{2}}\right) j_{3}^{(n)}\right]\left(\chi_{\lambda \mu o v}^{(n)}+\chi_{o \mu \lambda v}^{(n)}\right) \varepsilon_{\lambda \mu \nu} \hat{R}_{o} \\
\left.\quad-5\left(2 \beta \gamma^{(n)}+\alpha^{(n)}-5 \alpha^{(n)} \gamma^{(n) 2}\right) j_{3}^{(n)} \chi_{\lambda \mu \nu \pi}^{(n)} \varepsilon_{\mu \pi o} \hat{R}_{\lambda} \hat{R}_{v} \hat{R}_{o}\right\}
\end{aligned}
$$

expressed in a form which is substantially more compact, as well as more complete, than obtained previously. ${ }^{8}$ In the above expression, the parameters $\alpha^{(n)}, \beta$ and $\gamma^{(n)}$ embody the 
dependence on scattering geometry, being defined by:

$$
\begin{aligned}
\alpha^{(n)} & =\left(\boldsymbol{k} \cdot \boldsymbol{k}^{(n)}\right) \\
\beta & =\left(\boldsymbol{k} \cdot \boldsymbol{e}^{\prime}\right) \\
\gamma^{(n)} & =\left(\boldsymbol{k}^{(n)} \cdot \mathbf{e}^{\prime}\right)
\end{aligned}
$$

The above results offer a structure for the identification of ROA invariants by combining the results of experiments with different polarisation and beam geometries. It should be interesting to see whether this approach, despite its relative complexity, leads to results that are any more accurate than those based on more straightforward bondpolarisability arguments (see for example work by Barron et al. ${ }^{16}$ and Che and $\mathrm{Nafie}^{17}$ ). Nevertheless, it is perhaps more significant that the result for the intensity difference as expressed by eqn. (8) simplifies considerably in three special configurations, each of which is of experimental relevance. The first relates to study of the polarised circular differential $\Delta_{x}$, the second to forward scattering and the third to a backscattering geometry. In each of these cases $\beta$ and $\gamma^{(n)}$ both vanish identically, and as a consequence we obtain the following which holds for arbitrary group separation $R$ :

$$
\begin{aligned}
\Delta I \propto \sum_{n} \alpha^{(n)}\left[\left(j_{3}^{(n)}-4 j_{1}^{(n)}\right) \chi_{\lambda \mu \lambda \nu}^{(n)} \varepsilon_{\mu v o}^{(n)} \hat{R}_{o}+\left(j_{1}^{(n)}+j_{3}^{(n)}\right)\left(\chi_{\lambda \mu o v}^{(n)}+\chi_{o \mu \lambda v}^{(n)}\right)\right. \\
\left.\quad \times \varepsilon_{\lambda \mu v} \hat{R}_{o}-5 j_{3}^{(n)} \chi_{\lambda \mu v \pi}^{(n)} \varepsilon_{\mu \pi o} \hat{R}_{\lambda} \hat{R}_{v} \hat{R}_{o}\right]
\end{aligned}
$$

\section{Dependence on Group Separation}

To understand the detailed dependence of the above results, eqn. (8) and (12), on group separation, it is necessary to look at the behaviour, not only of the spherical Bessel functions, but also the coupling intrinsic in the molecular tensors $\chi_{i j k l}^{(n)}$. As discussed in the Appendix, the form of the inter-group coupling is normally such that $\chi_{i j k l}^{(6)}$ has a leading term of $R^{-6}$ dependence, while the other five molecular tensors have more significant $R^{-3}$ terms. The tensor $\chi_{i j k l}^{(1)}$ alone has an additional leading term independent of $R$, but this arises only in the case of coupling between a chemically equivalent pair. As far as the spherical Bessel functions are concerned, the short-range response is determined by the asymptotic limits $j_{1}^{(n)} \approx \frac{1}{3} k^{(n)} R$ and $j_{3}^{(n)} \approx \frac{1}{105} k^{(n)^{3}} R^{3}$, subject to satisfaction of the condition $k^{(n)} R \ll 1$.

By reference to eqn. (2) in which the wavevector parameters subsequently denoted by $\boldsymbol{k}^{(n)}$ appear explicitly in the six exponents, it is evident that for most scattering geometries the most stringent condition on short-range behaviour relates to the sixth and least significant term, for which $k^{(6)} R \ll 1$ if $R \ll 1 /|\Sigma \boldsymbol{k}|$. For the backscattering geometry the term with the most severe constraint is the first, where the linear approximation operates only for distances $R \ll 1 /|\Delta \boldsymbol{k}|$. It is also worth noting that under no circumstances will any $\boldsymbol{k}^{(\boldsymbol{n})}$ become a null vector. For example in the case of ROA generated by coupling between two equivalent groups in the forward scattering geometry, $k^{(1)} \equiv-\Delta \boldsymbol{k}$ has a modulus given by $\left(\omega-\omega^{\prime}\right) / c$ so that the $\chi_{i j k l}^{(1)}$ term which usually drives the circular differential scattering persists though only weakly. Previous treatments have, through the assumption $|\boldsymbol{k}| \approx\left|\boldsymbol{k}^{\prime}\right|$, led to the erroneous conclusion that such differential forward scattering, if driven by interference alone, would be forbidden. Within the short-range regime $R \ll \lambda / 2 \pi$, it is thus apparent from eqn. (A7) of the Appendix that for coupling between two identical groups in any scattering geometry, the celebrated linear dependence on group separation emerges. ${ }^{6}$ Under these circumstances vibrational excitation associated with symmetric and antisymmetric combination wavefunctions necessarily 
produces phase factors yielding a circular differential doublet with opposing signs, and consequently the term with linear dependence disappears if the doublet is not resolved. Differential scattering is then driven by interference between one-group and two-group scattering mechanisms in the same way as for coupling between two non-identical groups. For two inequivalent groups an inverse-square dependence operates in the short range. However in order to properly assess the extent of the short-range regime it is necessary to consider a factor that has in the past mostly been ignored, as is discussed in the following Section.

\section{Critique}

The present study has led to a reformulation of the theory of two-group Raman optical activity, with the distinction of accommodating the combined features of $(a)$ electronic wavefunction overlap; $(b)$ resonance response and (c) arbitrary orders of dipole-dipole coupling, also without a number of approximations that have hitherto been employed. The several features that characterise resonance response, in particular, are significant in the utilisation of resonance Raman studies in the characterisation of local chirality. A critical evaluation of the model nevertheless exposes certain shortcomings.

In general, a theoretical formulation in terms of coupling between just two groups is unnecessarily restrictive, though from the key eqn. (8) and (12) it is readily seen how coupling of a group, A, with any number of partners can be accommodated. Where coupling between dissimilar groups is involved, the result is quite simply a sum of the pairwise coupling terms, with each pair yielding six terms of precisely the form displayed in eqn. (8) and (12). For a set of equivalent groups, the same principle applies subject to employment of the appropriately symmetrised wavefunctions. These observations prompt the mention of one other distinct possibility, which is that the coupling considered to engender chiral discrimination in the Raman signal of any given group, A, need not necessarily be dominated by the nearest dissymmetrically placed chromophore, particularly if another nearby has an electronic absorption band near-resonant with the laser frequency. Indeed, under such circumstances the most significant coupling might easily be with a group five times more remote than the nearest.

One aspect of the coupling model that has certainly not received sufficient attention is the effect of the intervening electron distribution on the propagation of radiation between the two groups. This is a subject fraught with difficulty, and only a fully fledged calculation of molecular wavefunctions could properly address the problem. Of course if such an option were available one would not wish to resort to the crudeness of a twogroup model. Nonetheless it is at least possible to identify within the confines of the model some of the features that will reflect essentially refractive modifications to the distance dependence.

Clues to the nature of these refractive effects can be found through the observation that rate equations of a similar structure arise in the altogether different context of bimolecular photophysics. Here, processes of intermolecular energy transfer and bimolecular photoabsorption entail resonance-dipole coupling of exactly the same form as detailed in eqn. (A7). Not surprisingly, however, the closest parallel is with bimolecular Raman scattering. ${ }^{18}$ Recent work on such processes in terms of a polariton formulation has shown how the refractive index of the medium influences the form of the transition dipole interaction tensor when coupling over a distance of several molecular diameters is involved. Specifically, we find ${ }^{19-21}$

$$
V_{m n}(\omega, \boldsymbol{R}) \rightarrow \frac{1}{n_{\omega}^{2}}\left(\frac{n_{\omega}^{2}+2}{3}\right)^{2} V_{m n}\left(n_{\omega} \omega, \boldsymbol{R}\right)
$$


where the complex refractive index $n_{\omega}=n_{\omega}^{\prime}+\mathrm{i} n_{\omega}^{\prime \prime} \equiv \kappa_{\omega}^{1 / 2}$, and $\kappa_{\omega}$ is the relative permittivity. The imaginary part of $n_{\omega}$ represents a damping effect that can become particularly significant under resonance conditions. One other effect of the inter-group charge distribution can be found in the vectors $\boldsymbol{k}^{(\boldsymbol{n})}$ such as appear within the arguments of the spherical Bessel functions. As might be anticipated on a physical basis, the phase factors of the form $\exp \left(i k^{(n)} \cdot R\right)$ from which these originate in the six terms of eqn. (2) will be modified through $\boldsymbol{k} \rightarrow n_{\omega} \boldsymbol{k}, \boldsymbol{k}^{\prime} \rightarrow n_{\omega^{\prime}} \boldsymbol{k}^{\prime}$ by the refractive effect of the intervening electron distribution on optical wavelength. For completeness it should be noted that each term in the matrix element additionally carries the same multiplicative factor $\frac{1}{9} n_{\omega}^{-1 / 2} n_{\omega^{\prime}}^{1 / 2}\left(n_{\omega}^{2}\right.$ $+2)\left(n_{\omega^{\prime}}^{2}+2\right)$, but this has no bearing on the dependence on group separation.

It is instructive to consider the significance of these functions specifically in the present context of intramolecular coupling. Of course, no real meaning can be given to the concept of refractive index within a single molecule. Nevertheless it is clear that the intervening electron distribution will significantly modify the coupling tensor and the phase factors responsible for Raman optical activity. In other words the formulation of the model in terms of two groups separated by a vacuum is misleading, even when it is possible to argue a case for the distinct integrity of group wavefunctions. Assuming that the bulk refractive index, $n$, is at least a guide to the extent of radiative distortion between the two groups, we can surmise that the range of distances over which the linear or inverse-square distance dependence persists is rather less than might have been supposed, by a factor of the order of the refractive index. Whereas this might at first seem a rather insignificant matter, it is potentially very significant under the resonance conditions that are often favoured. Under such circumstances the retarded and radiative terms can no longer be ignored. What this means is that, whereas the principal features of a resonance study are associated with signal enhancement and a change in the selection rules, there is also a significant change in the distance dependence.

Two further points can be made. First, it is interesting to note that the distance dependence of the ROA associated with coupled but dissimilar groups involves mediation of the chiral influence of one group on a vibrational transition at the other by Förster-type radiationless energy transfer, a process that may itself be used to determine the distance between chromophore sites, based on its inverse-sixth-power distance dependence. ${ }^{22}$ Although the possible utilisation of ROA measurements as another kind of spectroscopic ruler is technically more demanding, it is a method that has the potential of yielding a great deal more information, and its inverse-square fall-off with distance makes it applicable over an appreciably larger range.

Finally, it may be observed that, whilst the results for the molecular tensors $\chi_{i j k l}^{(n)}$ as given by eqn. (A1)-(A6) of the Appendix apply subject to the specific preclusion of orbital overlap, a broadly similar structure can exist if orbital overlap is entertained. The appropriate formulation then necessitates a coupling model that seamlessly extends to distances below those where dipole interactions dominate. The prospect of such a theory has recently been advanced by a new formulation for electronic interactions and interchromophore excitation transfer. ${ }^{23}$ Although this work does not yet accommodate a vibrational structure for the electronic levels, it is a development that appears to offer considerable scope for the further advancement of two-group ROA.

\section{Appendix}

\section{A Structure for the $\chi_{i j k l}^{(n)}$ Tensors}

An explicit representation of the molecular response tensors $\chi_{i j k l}^{(n)}$ can be given by recourse to the assumption of a distinct integrity for the vibrational and electronic structures of the two groups involved. With the group coupling properly cast in terms of a retarded resonance dipole interaction we then have a set of results which, including 
terms of second order in the coupling tensor, is as follows:

$$
\begin{aligned}
& \chi_{i j k l}^{(1)}={ }^{\mathbf{A}} \alpha_{i j}^{\prime \mathbf{B}} \bar{\alpha}_{k l}^{\prime}+{ }^{\mathbf{A}} \beta_{i m j}^{\prime} V_{m n}(0, R)^{\mathbf{B}} \mu_{n}^{\mathbf{B}} \bar{\alpha}_{k l}^{\prime}+{ }^{\mathbf{A}} \beta_{i m j} V_{m n}\left(\omega-\omega^{\prime}, \boldsymbol{R}\right)^{\mathbf{B}} \mu_{n}^{\prime \mathbf{B}} \bar{\alpha}_{k l}^{\prime} \\
& +{ }^{\mathbf{A}} \alpha_{i j}^{\prime \mathbf{B}} \bar{\beta}_{k m l}^{\prime} \bar{V}_{m n}(0, R)^{\mathbf{A}} \bar{\mu}_{n}+{ }^{\mathbf{A}} \alpha_{i j}^{\prime \mathbf{B}} \bar{\beta}_{k m l} \bar{V}_{m n}\left(\omega-\omega^{\prime}, \boldsymbol{R}\right)^{\mathbf{A}} \bar{\mu}_{n}^{\prime} \\
& +{ }^{\mathbf{A}} \beta_{i m j}^{\prime} V_{m n}(0, R)^{\mathbf{B}} \mu_{n}^{\mathbf{B}} \bar{\beta}_{k o l}^{\prime} \bar{V}_{o p}(0, R)^{\mathbf{A}} \bar{\mu}_{p}+{ }^{\mathbf{A}} \beta_{i m j} V_{m n}\left(\omega-\omega^{\prime}, \boldsymbol{R}\right)^{\mathbf{B}} \mu_{n}^{\prime \mathbf{B}} \bar{\beta}_{k o l}^{\prime} \bar{V}_{o p}(0, \boldsymbol{R})^{\mathbf{A}} \bar{\mu}_{p} \\
& +{ }^{\mathbf{A}} \beta_{i m j}^{\prime} V_{m n}(0, R)^{\mathbf{B}} \mu_{n}^{\mathbf{B}} \bar{\beta}_{k o l} \bar{V}_{o p}\left(\omega-\omega^{\prime}, R\right)^{\mathbf{A}} \bar{\mu}_{p}^{\prime} \\
& +{ }^{\mathbf{A}} \beta_{i m j} V_{m n}\left(\omega-\omega^{\prime}, \boldsymbol{R}\right)^{\mathbf{B}} \mu_{n}^{\mathbf{B}} \bar{\beta}_{k o l} \bar{V}_{o p}\left(\omega-\omega^{\prime}, \boldsymbol{R}\right)^{\mathbf{A}} \bar{\mu}_{p}^{\prime}+\cdots \\
& \chi_{i j k l}^{(2)}={ }^{\mathbf{A}} \alpha_{i j}^{\prime \mathbf{B}} \bar{\alpha}_{k m} \bar{V}_{m n}\left(\omega^{\prime}, \boldsymbol{R}\right)^{\mathbf{A}} \bar{\alpha}_{n l}^{\prime}+{ }^{\mathbf{A}} \alpha_{i j}^{\prime \mathbf{B}} \bar{\alpha}_{k m}^{\prime} \bar{V}_{m n}(\omega, \boldsymbol{R})^{\mathbf{A}} \bar{\alpha}_{n l} \\
& +{ }^{\mathrm{A}} \beta_{i m j}^{\prime} V_{m n}(0, R)^{\mathbf{B}} \mu_{n}^{\mathbf{B}} \bar{\alpha}_{k o} \bar{V}_{o p}\left(\omega^{\prime}, \boldsymbol{R}\right)^{\mathrm{A}} \bar{\alpha}_{p l}^{\prime}+{ }^{\mathrm{A}} \beta_{i m j}^{\prime} V_{m n}(0, \boldsymbol{R})^{\mathbf{B}} \mu_{n}^{\mathrm{B}} \bar{\alpha}_{k o}^{\prime} \bar{V}_{o p}(\omega, \boldsymbol{R})^{\mathrm{A}} \bar{\alpha}_{p l} \\
& +{ }^{\mathbf{A}} \beta_{i m j} V_{m n}\left(\omega-\omega^{\prime}, \boldsymbol{R}\right)^{\mathbf{B}} \mu_{n}^{\prime \mathbf{B}} \bar{\alpha}_{k o} \bar{V}_{o p}\left(\omega^{\prime}, \boldsymbol{R}\right)^{\mathbf{A}} \alpha_{p l}^{\prime} \\
& +{ }^{\mathbf{A}} \beta_{i m j} V_{m n}\left(\omega-\omega^{\prime}, \boldsymbol{R}\right)^{\mathbf{B}} \mu_{n}^{\mathbf{B}} \bar{\alpha}_{k o}^{\prime} \bar{V}_{o p}(\omega, \boldsymbol{R})^{\mathbf{A}} \bar{\alpha}_{p l}+\cdots \\
& \chi_{i j k l}^{(3)}={ }^{\mathrm{A}} \alpha_{i j}^{\prime \mathrm{A}} \bar{\alpha}_{k m} \bar{V}_{m n}\left(\omega^{\prime}, \boldsymbol{R}\right)^{\mathbf{B}} \bar{\alpha}_{n l}^{\prime}+{ }^{\mathrm{A}} \alpha_{i j}^{\prime \mathbf{A}} \bar{\alpha}_{k m}^{\prime} \bar{V}_{m n}(\omega, \boldsymbol{R})^{\mathbf{B}} \bar{\alpha}_{n l} \\
& +{ }^{\mathbf{A}} \beta_{i m j}^{\prime} V_{m n}(0, R)^{\mathbf{B}} \mu_{n}^{\mathbf{A}} \bar{\alpha}_{k o} \bar{V}_{o p}\left(\omega^{\prime}, R\right)^{\mathbf{B}} \bar{\alpha}_{p l}^{\prime}+{ }^{\mathbf{A}} \beta_{i m j}^{\prime} V_{m n}(0, R)^{\mathbf{B}} \mu_{n}{ }^{\mathbf{A}} \bar{\alpha}_{k o}^{\prime} \bar{V}_{o p}(\omega, R)^{\mathbf{B}} \bar{\alpha}_{p l} \\
& +{ }^{\mathbf{A}} \beta_{i m j} V_{m n}\left(\omega-\omega^{\prime}, \boldsymbol{R}\right)^{\mathbf{B}} \mu_{n}^{\prime \mathbf{A}} \bar{\alpha}_{k o} \bar{V}_{o p}\left(\omega^{\prime}, \boldsymbol{R}\right)^{\mathbf{B}} \bar{\alpha}_{p l}^{\prime} \\
& +{ }^{\mathrm{A}} \beta_{i m j} V_{m n}\left(\omega-\omega^{\prime}, \boldsymbol{R}\right)^{\mathbf{B}} \mu_{n}^{\prime \mathbf{A}} \bar{\alpha}_{k o}^{\prime} \bar{V}_{o p}(\omega, \boldsymbol{R})^{\mathbf{B}} \bar{\alpha}_{p l}+\cdots \\
& \chi_{i j k l}^{(4)}={ }^{\mathbf{B}} \alpha_{i j}^{\prime \mathbf{B}} \bar{\alpha}_{k m} \bar{V}_{m n}\left(\omega^{\prime}, \boldsymbol{R}\right)^{\mathbf{A}} \bar{\alpha}_{n l}^{\prime}+{ }^{\mathbf{B}} \alpha_{i j}^{\prime \mathbf{B}} \bar{\alpha}_{k m}^{\prime} \bar{V}_{m n}(\omega, \boldsymbol{R})^{\mathbf{A}} \bar{\alpha}_{n l} \\
& +{ }^{\mathbf{B}} \beta_{i m j}^{\prime} V_{m n}(0, R)^{\mathbf{A}} \mu_{n}^{\mathbf{B}} \bar{\alpha}_{k o} \bar{V}_{o p}\left(\omega^{\prime}, \boldsymbol{R}\right)^{\mathbf{A}} \bar{\alpha}_{p l}^{\prime}+{ }^{\mathbf{B}} \beta_{i m j}^{\prime} V_{m n}(0, R)^{\mathbf{A}} \mu_{n}^{\mathbf{B}} \bar{\alpha}_{k o}^{\prime} \bar{V}_{o p}(\omega, \boldsymbol{R})^{\mathbf{A}} \bar{\alpha}_{p l} \\
& +{ }^{\mathbf{B}} \beta_{i m j} V_{m n}\left(\omega-\omega^{\prime}, \boldsymbol{R}\right)^{\mathbf{A}} \mu_{n}^{\prime \mathbf{B}} \bar{\alpha}_{k o} \bar{V}_{o p}\left(\omega^{\prime}, \boldsymbol{R}\right)^{\mathbf{A}} \bar{\alpha}_{p l}^{\prime} \\
& +{ }^{\mathbf{B}} \beta_{i m j} V_{m n}\left(\omega-\omega^{\prime}, \boldsymbol{R}\right)^{\mathbf{A}} \mu_{n}^{\mathbf{B}} \bar{\alpha}_{k o}^{\prime} \bar{V}_{o p}(\omega, \boldsymbol{R})^{\mathbf{A}} \bar{\alpha}_{p t}+\cdots \\
& \chi_{i j k l}^{(5)}={ }^{\mathrm{B}} \alpha_{i j}^{\prime \mathrm{A}} \bar{\alpha}_{k m} \bar{V}_{m n}\left(\omega^{\prime}, \boldsymbol{R}\right)^{\mathrm{B}} \bar{\alpha}_{n l}^{\prime}+{ }^{\mathrm{B}} \alpha_{i j}^{\prime \mathrm{A}} \bar{\alpha}_{k m}^{\prime} \bar{V}_{m n}(\omega, \boldsymbol{R})^{\mathrm{B}} \bar{\alpha}_{n l} \\
& +{ }^{\mathrm{B}} \beta_{i m j}^{\prime} V_{m n}(0, R)^{\mathrm{A}} \mu_{n}^{\mathrm{A}} \bar{\alpha}_{k o} \bar{V}_{o p}\left(\omega^{\prime}, \boldsymbol{R}\right)^{\mathrm{B}} \bar{\alpha}_{p l}^{\prime}+{ }^{\mathrm{B}} \beta_{i m j}^{\prime} V_{m n}(0, \boldsymbol{R})^{\mathbf{A}} \mu_{n}^{\mathrm{A}} \bar{\alpha}_{k o}^{\prime} \bar{V}_{o p}(\omega, \boldsymbol{R})^{\mathrm{B}} \bar{\alpha}_{p l} \\
& +{ }^{\mathrm{B}} \beta_{i m j} V_{m n}\left(\omega-\omega^{\prime}, \boldsymbol{R}\right)^{\mathbf{A}} \mu_{n}^{\prime \mathbf{A}} \bar{\alpha}_{k o} \bar{V}_{o p}\left(\omega^{\prime}, \boldsymbol{R}\right)^{\mathbf{B}} \bar{\alpha}_{p l}^{\prime} \\
& +{ }^{\mathrm{B}} \beta_{i m j} V_{m n}\left(\omega-\omega^{\prime}, \boldsymbol{R}\right)^{\mathbf{A}} \mu_{n}^{\prime \mathbf{A}} \bar{\alpha}_{k o}^{\prime} \bar{V}_{o p}(\omega, \boldsymbol{R})^{\mathbf{B}} \bar{\alpha}_{p l}+\cdots \\
& \chi_{i j k l}^{(6)}={ }^{\mathbf{B}} \alpha_{i m} V_{m n}\left(\omega^{\prime}, \boldsymbol{R}\right)^{\mathbf{A}} \alpha_{n j}^{\prime \mathbf{A}} \bar{\alpha}_{k o} \bar{V}_{o p}\left(\omega^{\prime}, \boldsymbol{R}\right)^{\mathbf{B}} \bar{\alpha}_{p l}^{\prime}+{ }^{\mathbf{B}} \alpha_{i m}^{\prime} V_{m n}(\omega, \boldsymbol{R})^{\mathbf{A}} \alpha_{n j}{ }^{\mathbf{A}} \bar{\alpha}_{k o} \bar{V}_{o p}\left(\omega^{\prime}, \boldsymbol{R}\right)^{\mathbf{B}} \bar{\alpha}_{p l}^{\prime} \\
& +{ }^{\mathbf{B}} \alpha_{i m}^{\prime} V_{m n}(\omega, \boldsymbol{R})^{\mathbf{A}} \alpha_{n j}{ }^{\mathbf{A}} \bar{\alpha}_{k o} \bar{V}_{o p}\left(\omega^{\prime}, \boldsymbol{R}\right)^{\mathbf{B}} \bar{\alpha}_{p l}^{\prime}+{ }^{\mathbf{B}} \alpha_{i m}^{\prime} V_{m n}(\omega, \boldsymbol{R})^{\mathbf{A}} \alpha_{n j}{ }^{\mathbf{A}} \bar{\alpha}_{k o}^{\prime} \bar{V}_{o p}(\omega, \boldsymbol{R})^{\mathbf{B}} \bar{\alpha}_{p l}+\cdots
\end{aligned}
$$

Note that the prime on any such tensor designates the vibrational transition, and that the formal selection rules governing the $\mu^{\prime}, \alpha^{\prime}$ and $\beta^{\prime}$ tensors are those that normally apply to IR absorption, Raman scattering and hyper-Raman scattering, respectively. The involvement of non-Raman selection rules essentially reflects a lowering of local symmetry through the inter-group coupling. The retarded resonance dipole coupling tensor $\boldsymbol{V}$ featured in eqn. (A1)-(A6) is given by:

$$
\begin{aligned}
V_{m n}(\omega, \boldsymbol{R}) & =\frac{\mathrm{i} \omega^{3}}{12 \pi \varepsilon_{0} c^{3}}\left[\left(\delta_{m n}-3 \hat{R}_{m} \hat{R}_{n}\right) h_{2}^{(1)}(\omega R / c)-2 \delta_{m n} h_{0}^{(1)}(\omega R / c)\right] \\
& =\frac{\omega^{3} \exp (\mathrm{i} \omega R / c)}{4 \pi \varepsilon_{0} c^{3}}\left[\left(\delta_{m n}-3 \hat{R}_{m} \hat{R}_{n}\right)\left(\frac{c^{3}}{\omega^{3} R^{3}}-\frac{\mathrm{i} c^{2}}{\omega^{2} R^{2}}\right)-\left(\delta_{m n}-\hat{R}_{m} \hat{R}_{n}\right) \frac{c}{\omega R}\right]
\end{aligned}
$$


where the functions $h_{0}^{(1)}$ and $h_{2}^{(1)}$ are spherical Bessel functions of the third kind. ${ }^{15}$ For Stokes Raman scattering, short-range asymptotic behaviour $\boldsymbol{V} \propto R^{-3}$ operates for all couplings over distances $R \ll c / \omega \equiv \lambda / 2 \pi$, a condition that is normally satisfied.

From eqn. (A1)-(A6) it is evident that $\chi^{(1)}$ has a leading term not involving the dipole coupling tensor $\boldsymbol{V} ; \chi^{(2-5)}$ have leading terms involving $\boldsymbol{V}$ linearly, whilst the leading term of $\chi^{(6)}$ is quadratic in $\boldsymbol{V}$ and is therefore relatively insignificant. The imaginary part of the coupling tensor contributes to the ROA where resonance conditions allow the imaginary part of the scattering tensors to become significant, and it should therefore be omitted only in calculations dealing with off-resonance behaviour. In the single case of the $\chi^{(6)}$ term, the imaginary part of $\boldsymbol{V}$ can play a role even under non-resonant conditions. It may also be noted that near to resonance, damping modifications to the coupling tensor lead to the emergence of a real contribution from the part that for coupling in vacuo is entirely imaginary (see Section 4).

The general formulation above accommodates cases where either molecule A or molecule B is excited by the overall Raman process. If A and B are chemically dissimilar, only the terms with excitation referred to the species producing the Raman signal apply. Consequently the five terms involving $\chi^{(1)}-\chi^{(5)}$ are on a similar footing, each being driven by terms linear in $\boldsymbol{V}$. As such, the origin of the chiral discrimination may be understood as an interference between single-centre scattering and two-group scattering mediated by dipole-dipole coupling. In a different context, the latter is responsible for the $R^{-6}$ dependence of Förster energy transfer; consequently the interference generates a short-range $R^{-3}$ dependence of the molecular tensors. The full set of terms has to be employed when A and B are structurally identical, and in such a case the vibrational wavefunctions of the two groups inevitably interfere to the extent that only their symmetrised and antisymmetrised combinations are stationary states for the system. Consequently the above expressions for the $\chi_{i j k l}^{(n)}$ tensors have to be read with regard to a combination phase that may be regarded as incorporated in the transition tensors $\boldsymbol{\mu}^{\prime}, \mathbf{a}^{\prime}$ and $\boldsymbol{\beta}^{\prime}$.

\section{References}

1 M. Born, Phys. Zeit., 1915, 16, 251.

2 C. W. Oseen, Ann. Phys., 1915, 48, 1.

3 W. Kuhn, Trans. Faraday Soc., 1930, 26, 293.

4 S. F. Boys, Proc. R. Soc. London A, 1934, 144, 655.

5 J. G. Kirkwood, J. Chem. Phys., 1937, 5, 479.

6 L. D. Barron and A. D. Buckingham, J. Am. Chem. Soc., 1974, 96, 4769.

7 A. J. Stone, Mol. Phys., 1977, 33, 293.

8 D. L. Andrews and T. Thirunamachandran, Proc. R. Soc. London A, 1977, 358, 311.

9 A. Gohin and M. Moscovits, J. Am. Chem. Soc., 1981, 103, 1660.

10 V. A. Morozov, J. Raman Spectrosc., 1993, 24, 585.

11 L. D. Barron and L. Hecht, in Circular Dichroism-Principles and Applications, ed. K. Nakanishi, N. Berova and R. W. Woody, VCH, New York, 1994, p. 179.

12 P. L. Polavarapu, J. Phys. Chem., 1990, 94, 8106.

13 D. P. Craig and T. Thirunamachandran, Molecular Quantum Electrodynamics, Academic, London, 1984 , p. 188.

14 D. L. Andrews and M. J. Harlow, Phys. Rev. A, 1984, 29, 2796.

15 Pocketbook of Mathematical Functions, ed. M. Abramowitz and I. A. Stegun, Verlag Harri Deutsch, Thun, 1984, p. 154.

16 L. D. Barron, J. R. Escribano and J. F. Torrance, Mol. Phys., 1986, 57, 653.

17 D. Che and L. A. Nafie, Chem. Phys. Lett., 1992, 189, 35.

18 D. L. Andrews and N. P. Blake, Phys. Rev. A, 1990, 41, 2547.

19 D. L. Andrews and G. Juzeliūnas, J. Lumin., 1994, 60, 834.

20 G. Juzeliūnas and D. L. Andrews, Phys. Rev. B, 1994, 49, 8751.

21 D. L. Andrews and G. Juzeliūnas, work in progress.

22 G. J. Liu, Macromolecules, 1993, 26, 1144.

23 G. D. Scholes and K. P. Ghiggino, J. Phys. Chem., 1994, 98, 4580. 